\title{
Using of the Generalized Special Relativity in Deriving the Equation of the Gravitational Red-Shift
}

\author{
Mahmoud Hamid Mahmoud Hilo ${ }^{1,2}$ \\ ${ }^{1}$ Department of Physics, Faculty of Education, Al-Zaiem Al-Azhari University, Omdurman, Sudan \\ ${ }^{2}$ Department of Physics, Faculty of Science and Arts at Al-Rass, Qassim University, KSA \\ E-mail:mhhlo@qu.edu.sa \\ Received January 9, 2011; revised March 10, 2011; accepted March 21, 2011
}

\begin{abstract}
In this work we present a study of a new method to prove the equation of the gravitational red shift of spectral lines. That's according to the generalized special relativity theory. The equation of the gravitational red shift of spectral lines has been studied in many different works, using different methods depending on the Newtonian mechanics, and other theories. Although attention was drawn to the fact that the well-known expression of the gravitational Red-Shift of spectral lines may be derived with no recourse to the general relativity theory! In this study a unique derivation has been done using the Generalized Special Relativity (GSR) and the same result obtained.
\end{abstract}

Keywords: Generalized, Red-Shift, GSR, Approximations, Gravitational

\section{Introduction}

In physics, light or other forms of electromagnetic radiations of a certain wavelength originating from a source placed in a region of strong gravitational field (and which could be said to have climbed "uphill" out of the gravity well) will be found to be longer wavelength when received by observer in a region of weak gravitational field [1]. If we apply to optical wave-lengths this manifests itself as a change in the color of the light, the wavelength is shifted towards the red (making it less energetic, longer in wavelength, and lower in frequency) part of the spectrum. This effect is called the gravitational red shift, and the other spectral lines found in the light, will also be shifted towards the longer wavelength, or red end of the spectrum. This shift can be observed along the entire electromagnetic spectrum [2].

In all basic studies involved in the theory of general relativity, attention was drawn to the three main problems related to it, those are Well-Known advance of the perihelion of the planet Mercury, the Gravitational Deflection of Light Rays and the Gravitational Red-Shift of Spectral Lines. The gravitational red-shift discussed within the emitted rays from a particle that located in the field of another rest particle due to a spherical symmetry (such as Solar field), the atoms that compose the gases edges of a rest star forms light sources in the star field, and ac- cording to this information the Gravitational Red-Shift obtained [3]. This study introduces a new method to obtain the same result of the Gravitational Red-Shift using the Generalized Special Relativity theory, (GSR) by adopting the approximation of the gravitational potential.

\section{Objective}

Objective of this work is to prove the equation of the gravitational redshift as well as to test the theory of the generalized special relativity.

\section{Generlized Special Relativity (GSR) Theory}

The Generalized Special Relativity theory is a new form of the special relativity theory that adopts the gravitational potential, and it gives the formula of relative mass to be as follows [4]:

$$
m=\frac{g_{00} m_{0}}{\sqrt{g_{00}-\frac{\mathrm{v}^{2}}{c^{2}}}}
$$

where, $g_{00}=1+\frac{2 \phi}{c^{2}}$ and $\varphi$ denotes the gravitational potential, or the field in which the mass is measured.

The derivation of the mass Equation 1 using the Gen- 
eralized Special Relativity (GSR) can be found as follows:

In the Special Relativity (SR), the time, length and mass can be obtained in any moving frame by either multiplying or dividing their values in the rest frame by a factor $\gamma$.

$$
\gamma=\sqrt{1-\frac{v^{2}}{c^{2}}}
$$

where $c$ is the speed of light, and $v$ is the velocity of the particle.

To see how gravity affects these quantities it is a convenient to re-express $\gamma$ in terms of the proper time, [5]:

$$
c^{2} \mathrm{~d} \tau^{2}=\boldsymbol{g}_{\mu \nu} \mathrm{d} x^{\mu} \mathrm{d} x^{\nu}
$$

where $\boldsymbol{g}_{\mu \nu}$ is the metric tensor, and, $\boldsymbol{\mu}$ and $\boldsymbol{v}$ denotes the contravariant (covariant) vectors.

Which is a common language to both Special Relativity (SR) and General Relativity (GR). We know that in special relativity (SR) Equation 3 reduces to [6]:

$$
c^{2} \mathrm{~d} \tau^{2}=c^{2} \mathrm{~d} t^{2}-\mathrm{d} x^{i} \mathrm{~d} x^{i}, x^{0}=c . t
$$

where $i$ denotes the particle position (covariant) vector according to Lorentz covariance.

Then

$$
\frac{\mathrm{d} \tau}{\mathrm{d} t}=\sqrt{1-\frac{1}{c^{2}} \times \frac{\mathrm{d} x^{i}}{\mathrm{~d} t} \times \frac{\mathrm{d} x^{i}}{\mathrm{~d} t}}=\sqrt{1-\frac{v^{2}}{c^{2}}}=\gamma
$$

Thus we can easily generalize $\gamma$ to include the effect of gravitation by using Equation 3 and by adopting the weak field approximation where [6]:

$$
g_{11}=g_{22}=g_{33}=-1, g_{00}=1+\frac{2 \phi}{c^{2}}
$$

Then $\gamma$ becomes

$$
\gamma=\frac{\mathrm{d} \tau}{\mathrm{d} t}=\sqrt{g_{00}-\frac{1}{c^{2}} \times \frac{\mathrm{d} x^{i}}{\mathrm{~d} t} \times \frac{\mathrm{d} x^{i}}{\mathrm{~d} t}}=\sqrt{g_{00}-\frac{v^{2}}{c^{2}}}
$$

When the effect of motion only is considered, the expression for time in special relativity (SR) takes the form [5].

$$
\mathrm{d} t=\frac{\mathrm{d} t_{0}}{\sqrt{1-\frac{v^{2}}{c^{2}}}}
$$

where the subscript 0 stands for the quantity measured in the rest frame. While gravity only affects time, its expression is given by [6].

$$
\mathrm{d} t=\frac{\mathrm{d} t_{0}}{\sqrt{g_{00}}}
$$

In view of Equations 7 and 8 , the expression:

$$
\mathrm{d} t=\frac{\mathrm{d} t_{0}}{\gamma}
$$

can be generalized to recognize the effect of motion as well as gravity on time, to get:

$$
\mathrm{d} t=\frac{\mathrm{d} t_{0}}{\sqrt{g_{00}-\frac{v^{2}}{c^{2}}}}
$$

The same result can be obtained for the volume where the effect of motion and gravity respectively gives [6]:

$$
\begin{aligned}
& V=V_{0} \sqrt{1-\frac{v^{2}}{c^{2}}} \\
& V=\sqrt{g} V_{0}=\sqrt{g_{00}} V_{0}
\end{aligned}
$$

The generalization can be done by utilizing Equation 7 to find that:

$$
V=\gamma V_{0}=\sqrt{g_{00}-\frac{v^{2}}{c^{2}}} \cdot V_{0}
$$

To generalize the concept of mass to include the effect of gravitation we use the expression for the Hamiltonian in general relativity, i.e. [6]:

$$
H=\rho c^{2}=g_{00} T^{00}=g_{00} \rho_{0}\left(\frac{\mathrm{d} x^{0}}{\mathrm{~d} \tau}\right)^{2}=g_{00} \frac{\rho_{0} c^{2}}{\gamma^{2}}=g_{00} \frac{m_{0} c^{2}}{V_{0} \gamma^{2}}
$$

where $H$ is Hamiltonian, $\rho$ is the density, and $T^{00}$ is energy tensor.

Using Equations 14 and 15 yields:

$$
\rho c^{2}=\frac{m c^{2}}{V}=\frac{g_{00} m_{0} c^{2}}{\gamma V}
$$

Therefore:

$$
m=\frac{g_{00} m_{0}}{\sqrt{g_{00}-\frac{v^{2}}{c^{2}}}}
$$

which is the expression of mass in the presence of gravitation.

In view of Equation 1 and when we substitute the value of $g_{00}$, then the relative mass according to (GSR) is found to be:

$$
m=\frac{m_{0}\left(1+\frac{2 \varphi}{c^{2}}\right)}{\sqrt{1+\frac{2 \phi}{c^{2}}-\frac{v^{2}}{c^{2}}}}
$$

When the field is weak in the sense that:

$$
\frac{2 \varphi}{c^{2}} \ll 1
$$

And when the speed $v$ is very low such that:

$$
\frac{v^{2}}{c^{2}} \ll 1
$$


Equation 18 reduced to:

$$
m=\frac{m_{0}\left(1+\frac{2 \varphi}{c^{2}}\right)}{\sqrt{1+\frac{2 \varphi}{c^{2}}}}=m=m_{0} \sqrt{\left(1+\frac{2 \varphi}{c^{2}}\right)}
$$

Using the identity $(1+x)^{n}=1+n \cdot x$ for $x \ll 1$

We can get that

$$
m=m_{0}\left(1+\frac{\varphi}{c^{2}}\right)
$$

And, when the field is so strong such that

$$
\frac{2 \varphi}{c^{2}} \gg 1 \text { and } \frac{v^{2}}{c^{2}} \ll 1
$$

Then Equation 18 reduced to

$$
m=m_{0} \sqrt{\frac{2 \varphi}{c^{2}}}
$$

\section{Derivation of the Red Shift Equation}

Einstein's mass and energy equivalent relation, agrees that the energy of a particle is given by [7]:

$$
E=m c^{2}=h \cdot v
$$

where $h$ is Planck's constant and $c$ is the speed of light, substitute the mass $m$ from Equation 22 in Equation 25, one gets:

$$
E=m c^{2}=m_{0} c^{2}\left(1+\frac{\varphi}{c^{2}}\right)
$$

The difference in particles or light energy presented as [7].

$$
\Delta E=m c^{2}-m_{0} c^{2}=m_{0} c^{2}\left(1+\frac{\varphi}{c^{2}}\right)-m_{0} c^{2}=m_{0} \phi
$$

Then

$$
\frac{\Delta E}{E_{0}}=\frac{\Delta h v}{h v_{0}}=\frac{m_{0} \varphi}{m_{0} c^{2}}=\frac{\varphi}{c^{2}}
$$

Or

$$
\frac{\Delta v}{v_{0}}=\frac{\varphi}{c^{2}}
$$

As mentioned before, $\varphi$ denotes the potential field, which can be given by

$$
\varphi=\frac{-M G}{r}
$$

Substituting $\varphi$ in Equation 29, then the following equation is obtained

$$
\frac{\Delta v}{v_{0}}=\frac{\varphi}{c^{2}}=\frac{-M G}{r c^{2}}
$$

Equation 30 is found to be the same formula of the Gravitational Red-Shift of the spectral lines which is the expected result.

\section{Materials and Methods}

Materials and methods applied in this paper are only concentrated on mathematical derivation of the gravitational redshift equation. Starting with the equation of mass in the sense of the generalized special relativity theory, we found that it is possible to prove gravitational redshift equation.

\section{Results}

Equations 17 and 24 are obtained to give the expression of mass in the presence of gravitational field within the special relativity theory, which means generalizing the special relativity to include the gravitational potential.

Equation 30 is the same as the equation of the gravitational red-shift, obtained by Equation 24 using Einstein's mass and energy equivalent relation, and that presents the generalized special relativity as an adequate theory in proving the equations of famous physical phenomena.

\section{Discussion}

The expression of gravitational red shift from the generalized special relativity (GSR) model stand point, was derived in previous Section 4, the expression obtained in Equation 30 is in complete agreement with that obtained from general relativity in [7]. In one hand, that means adopting of the weak field approximation in the generalized special relativity, leads to succeed in proving an important theory in physics such as the gravitational redshift of the spectral lines, and on the other hand, it explains that the appearance of the weak field does not affect the red shift negatively, but, oppositely the generalized special relativity succeeded again.

The result obtained in this study using Generalized Special Relativity theory (GSR), and the result obtained by Evans and Dunning [7], which, used the Newtonian mechanics, agree that the (GSR) is another crucial theory to examine one of the three important tests related to the theory of the general relativity, those are Well-Known advance of the perihelion of the planet Mercury, the Gravitational Deflection of Light Rays and the Gravitational Red-Shift of Spectral Lines [6,8,9].

When we used the approximation of $g_{00}$, we depend completely on the result found in [5], dealing with the special relativity (SR) in the presence of gravitational potential and not only on the general relativity (GR). 


\section{References}

[1] G. F. Lewis, et al. "Cosmological Radar Ranging in an Expanding Universe," Monthly Notices of the Royal Astronomical Society, Vol. 388, No. 3, 2008, pp. 960-964. doi:10.1111/j.1365-2966.2008.13477.x

[2] T. Koupelis and K. F. Kuhn, "In Quest of the Universe," 5th Edition, Jones \& Bartlett Publishers, Sudbury, 2007, p. 557.

[3] O. D. Jefimenko, "Electromagnetic Retadration, and Theory and Relativity," 2nd Edition, 2005.

[4] M. H. M. Hilo, et al. "Using of the Generalized Special Relativity in Estimating the Proton (Nucleon) Mass to Explain the Mass Defect," Natural Science, Vol. 3, No. 2, pp. 141-144. doi:10.4236/ns.2011.32020

[5] F. L. Derek, "An Introduction to Tensor Calculus and Relativity," 3rd Edition, John Wiley \& Sons Ltd., New York, 1982, pp. 5-6.

[6] S. Weinberg, "Gravitation and Cosmology," 3rd Edition, John Wiley \& Sons Ltd., New York, 1972, p. 688.

[7] R. F. Evans and J. Dunning-Davies, "The Gravitational Red-Shift," University of Hull, England, 2004, p. 3.

[8] R. C. Tolman, "Relativity, Thermodynamics and Cosmology," 1st Edition, Oxford University Press, Oxford, 1934, p. 591.

[9] R. Adler, M. Bazin and M. Schiffer, "Introduction to General Relativity," 2nd Edition, McGraw-Hill, New York, 1975. 